\title{
O PAPEL DO PROFISSIONAL DE EDUCAÇÃO FÍSICA NA SAÚDE PÚBLICA: ANÁLISES E REFLEXÕES A PARTIR DE REVISÕES BIBLIOGRÁFICAS ${ }^{* 1}$
}

\author{
Danielle Oliveira Freitas ${ }^{2}$ \\ Evandro Salvador Alves de Oliveira ${ }^{3}$
}

\section{Introdução}

Esta pesquisa é fruto de um trabalho de conclusão de curso (Educação Física) construído com base nos pressupostos do método qualitativo. A pesquisa é de natureza bibliográfica e, aqui, o foco das discussões está em torno da temática que envolve o papel do profissional de Educação Física no campo da saúde pública.

A construção deste trabalho ocorreu por meio de algumas motivações, principalmente pelo interesse em conhecer as possibilidades de atuação do profissional com formação em Educação Física (modalidade bacharelado), considerando suas várias áreas de atuação, sobretudo na área da saúde, visto que a maior demanda de profissionais está no campo das áreas fitness e da competição/rendimento.

Sendo assim, esta pesquisa foi construída a partir de objetivos previamente delineados, de maneira a permitir que um considerável avanço sobre as discussões que envolvem esta temática pudesse acontecer. Para isso, recorremos à literatura para buscar e encontrar possíveis respostas que pudessem responder o problema desta pesquisa - que consistiu saber: o que dizem os textos científicos sobre o papel do profissional de Educação Física no campo da saúde pública, considerando os últimos dez anos de produção? Para responder ao problema traçamos al-

* 10.29388/978-65-86678-46-8-0-f.129-144

${ }^{1} \mathrm{O}$ presente capítulo é fruto de um trabalho de conclusão de curso (Bacharel em Educação Física - UNIFIMES, GO) orientado pelo Prof. Dr. Evandro Salvador Alves de Oliveira.

${ }^{2}$ Graduada em Educação Física pela UNIFIMES. Especialização (em andamento) em Anatomia Humana com Ênfase em Dissecação pelo CEUSG - Centro de Ensino Unificado do Sudoeste Goiano.

${ }^{3}$ Doutor em Estudos da Criança pela Universidade do Minho - Portugal. Doutor em Educação pela Universidade de Uberaba. Pesquisador e Docente do curso de Educação Física da UNIFIMES. 
guns objetivos, tais como: analisar as produções bibliográficas sobre o papel do profissional de Educação Física na Saúde Pública dos últimos dez anos (2009-2019) - objetivo nuclear.

E os objetivos específicos foram: abordar as possibilidades de intervenção do profissional de Educação Física na sociedade; explanar o tema saúde pública no Brasil; e discutir os principais assuntos abordados nas publicações que se referem à inserção/atuação do profissional de Educação Física na saúde pública.

A metodologia utilizada para a elaboração deste trabalho foi essencialmente a de abordagem qualitativa, que trata de analisar aspectos que se distanciam de perspectivas quantitativas, numéricas e etc. Foram utilizados alguns bancos de dados, com intuito de coletar dados (trabalhos científicos), como: Scielo e Lilacs - dos quais foram selecionadas publicações no recorte temporal de dez anos, utilizando os seguintes descritores para a pesquisa: profissional de educação física e saúde pública.

Das publicações encontradas, foram lidos os resumos e descartados os trabalhos que se distanciavam do objeto de estudo. Após filtragem, treze (13) publicações foram consideradas elegíveis, porque tratavam especificamente da atuação/inserção do profissional de Educação Física no âmbito da Saúde Pública no Brasil.

\section{Intervenções do profissional de educação física: multipli- cidades}

A formação em Educação Física tem duas identidades distintas, conforme apontam Nunes, Votre e Santos (2012), ao caracterizá-las em: formação para a atuação do professor de Educação Física, na educação básica; e em formação para o bacharel, no mundo do trabalho não escolar. Aqui daremos ênfase às formas de atuação do bacharel em Educação Física, visto que é este o profissional que atua na área da saúde.

São várias as possibilidades de intervenção do profissional de Educação Física na sociedade. Este profissional é capacitado para atuar com diversos públicos, desde a fase de desenvolvimento infantil até o envelhecimento.

Salles, Faria e Nascimento (2015) destacam como principais contextos habituais de intervenção do profissional de Educação Física as academias de ginástica, academias de musculação e clubes desportivos, e como ambientes não tradicionais os hospitais, as clínicas de reabilitação, 
os grupos de terceira idade e as equipes multiprofissionais (como os Núcleos de Atenção à Saúde da Família - NASFs).

A formação de bacharel habilita o profissional de Educação Física a realizar avaliações físicas, prescrever exercícios e treinos com os mais variados objetivos, além de ensinar, acompanhar e corrigir a execução dos movimentos, considerando os aspectos fisiológicos, biomecânicos e psicológicos de cada indivíduo. Os atendimentos podem ser feitos individualmente ou em coletivo, e sempre deve ser considerada a especificidade ou individualidade biológica de cada um, além dos aspectos sociais de seu cotidiano.

É de amplo conhecimento que a prática regular de atividade física é considerada um fator determinante na condição de saúde dos indivíduos, e cabe ao profissional de Educação Física incentivar esta prática, conscientizando os grupos populacionais de seus benefícios. Não cabe aqui, destacar quais são os benefícios da atividade na vida do homem. Sabemos que são inúmeros, mas procuraremos focar em nosso objeto de estudo, de maneira a buscar respostas para o problema e objetivos.

\section{Crianças e Atividade Física}

Fernandes et al. (2017) analisaram a relação entre o desenvolvimento motor e a prática de atividade física com crianças em idade escolar e identificaram que a prática regular contribui significativamente para o desenvolvimento das habilidades motoras básicas fundamentais das crianças.

Por outro lado, Gallahue (2013) observou que a prática regular de atividade física é capaz de estimular a mineralização óssea e o desenvolvimento muscular, além de ajudar a retardar a formação de depósitos de gordura, auxiliando no desenvolvimento motor das crianças. Trata-se de uma importante referência que se dedica a estudar o desenvolvimento motor de bebês e crianças na cultura contemporânea.

Além de auxiliar no desempenho motor das crianças, a prática regular de atividades físicas pode melhorar, também, o desenvolvimento cognitivo, conforme verificado por Merege Filho et al (2013). Os autores, após avaliarem o desempenho cognitivo de crianças saudáveis através do Teste de Memória, perceberam uma influência positiva da atividade física de lazer sobre a memória incidental dessas crianças, sugerindo que crianças que se envolvem em atividades físicas esportivas no tempo de lazer com mais frequência, conseguem maior desempenho na memória de curto prazo. 


\section{Gestantes e Atividade Física}

Durante a gestação ocorrem mudanças fisiológicas no corpo da mulher. Aires (2018), por exemplo, destaca alguns parâmetros funcionais que apresentam alteração encontrando-se aumentados durante a gestação: ventilação, retenção de água e eletrólitos, metabolismo basal (há maior consumo de energia), volume sanguíneo, dentre outros. O significativo aumento de peso corporal também é comum durante a gravidez (em média de 10 a $12 \mathrm{~kg}$ ).

Além desse aspecto, Barret (2014) afirma que, particularmente durante a gestação, os ovários secretam um hormônio chamado relaxina, que tem como função relaxar os ligamentos da sínfise púbica e o colo uterino para facilitar o nascimento do feto, podendo provocar desconforto na região lombar.

Em outro estudo, publicado por Silva e Lucena (2014), há a constatação de que a prática de exercício físico regular e bem orientada promove essenciais benefícios que trazem segurança e uma qualidade de vida melhor, tanto para a mãe quanto para o feto. Para exemplificar, destacamos os achados de Souza, Vieira e Neto (2018). Os dados de seus estudos mostraram que a prática regular de atividade física promove benefícios para as gestantes tais como: controle da obesidade, diminuição de dores na lombar, menor chance de incontinência urinária, melhora da frequência cardíaca e melhora da estabilidade muscular. Tais benefícios podem favorecer a gestação, o parto e o retorno às condições ponderais do pós-parto.

É necessário, portanto, o acompanhamento do profissional de Educação Física que reconhece quais os tipos de exercícios mais adequados e em quais proporções podem ser realizados. Nesse aspecto, Nascimento et al. (2014) enfatizaram, ainda, a importância do acompanhamento de equipe multidisciplinar que permita uma abordagem global da saúde da gestante.

\section{Obesos e Atividade Física}

Vivolo e Sarno (2015) destacam que, tanto no Brasil como na maioria dos países, o excesso de gordura corporal é um dos mais importantes problemas de saúde pública, por conta dos custos diretos e indiretos associados às consequências físicas, psíquicas, sociais e econômicas causados pela obesidade. 
Várias doenças podem estar associadas à obesidade. Mancini (2015) analisou vários estudos e listou as seguintes patologias: Diabetes mellitus tipo 2, síndrome metabólica, neoplasias, osteoartrose, pancreatite aguda, doença hepática gordurosa não alcoólica, doenças respiratórias, doença do refluxo gastresofágico, asma brônquica, insuficiência renal crônica, doença hemorroidária, infertilidade masculina e feminina, síndrome dos ovários policísticos, disfunção erétil, disfunção cognitiva e demência.

As contribuições da dieta e da inatividade física são evidentes no aumento de massa adiposa corporal. Dâmaso e Sanches (2015), reconhecem que a prática de exercícios físicos regulares é uma boa estratégia para o emagrecimento de pessoas obesas, pois é o principal fator responsável pelo aumento do gasto calórico que favorece o balanço energético negativo e apresenta uma correlação inversa à ocorrência de sérias comorbidades, que variam com a idade e o grau da obesidade.

A prescrição do exercício físico para obesos deve ser feita por profissional de Educação Física e acompanhado por equipe multidisciplinar. Dâmaso e Sanches (2015, p. 469) enfatizam que o exercício deve ser adaptado de acordo com o grau de obesidade, a idade e a presença de comorbidades e fatores de risco associados. $\mathrm{O}$ incentivo à adoção de um estilo de vida mais saudável e ativo também é essencial.

\section{Idosos e Atividade Física}

O envelhecimento é um processo natural e inevitável. Assis (2012) afirma que a forma como a pessoa lida com as mudanças no processo de envelhecimento repercute inevitavelmente na relação que ela estabelece com o seu próprio corpo e saúde.

A literatura mostra que a prática regular de atividades físicas auxilia no desempenho funcional de idosos além de melhorar seu bem-estar psicológico. Fernandes et al (2012) confirmaram isso em um estudo onde aplicaram um programa de exercícios físicos direcionados para treino de força, equilíbrio e propriocepção e concluíram que tal prática foi capaz de melhorar o desempenho físico e funcional dos idosos.

\section{Pessoas com necessidades especiais}

De acordo com Pedrinelli e Verenguer (2013, p. 12), “os direitos sociais asseguram o direito de igual oportunidade, independentemente da 
condição diferente e peculiar que uma pessoa possa apresentar". Os autores afirmam, ainda, que as organizações esportivas, de dança, de jogos e de ginástica tem contribuído de forma significativa para ampliar possiblidades para a participação de pessoas com necessidades especiais.

Neste contexto, Greguol e Costa (2013) organizaram uma série de achados sobre a influência da atividade física e a forma de se inserir essa prática no cotidiano de pessoas com necessidades especiais. Dentre os públicos estudados, destacam-se as seguintes condições: deficiência visual, deficiência intelectual, deficiência auditiva, lesão da medula espinhal, amputações e anomalias congênitas, distúrbios neurológicos e musculares, condições ortopédicas limitantes, osteoporose, distúrbios posturais, distúrbios respiratórios (asma), prevenção e reabilitação cardíaca e saúde mental.

Em todos os grupos, com a prática regular de atividades físicas, observou-se melhora na capacidade funcional, melhora no condicionamento físico, na força, na flexibilidade, além dos benefícios psicológicos que promovem o bem-estar desses indivíduos. Outro ponto positivo da prática regular de atividades físicas por esses grupos que necessitam de cuidados especiais, é a sua (re)inserção na sociedade.

Além das possibilidades de intervenção do profissional de Educação Física nos diversos grupos da sociedade, há de se considerar também, sua atuação na preparação física de atletas e no condicionamento necessário para determinadas atividades cotidianas.

\section{Saúde pública no Brasil: um breve panorama histórico}

No Brasil, o avanço da saúde pública teve início com movimentos sociais que marcaram anos de luta. Tal afirmação parte das considerações registradas por Carvalho (2013), que realizou uma análise documental histórica na qual evidenciou que o atual sistema público de saúde resultou de décadas de luta de um movimento que se denominou Reforma Sanitária.

Santos (2013), observou em seus estudos que antes da Constituição de 1988, não havia o reconhecimento de diversos direitos fundamentais ao indivíduo - entre eles o da saúde - que era garantido, apenas, no que se referia à prevenção de doenças (como vacinação e serviços de pronto-socorro, além de vigilância em saúde). Outras demandas de saúde advindas da população eram sanadas, em partes, pela assistência social e serviços previdenciários, que beneficiavam somente trabalhadores com registro em carteira de trabalho e seus dependentes. Segue a comparação 
das constituições anteriores à de 1988, feitas por Santos (2013), onde são apontados o que nelas constava sobre saúde.

Com a criação do Sistema Único de Saúde - SUS, os brasileiros passaram a ter acesso aos serviços de saúde no que tange à atenção integral - não somente assistencial - conforme evidencia a literatura. O SUS é, portanto, concebido como o conjunto de ações e serviços de saúde assim como afirma Rosemberg (2013) -, prestados por órgãos e instituições públicas federais, estaduais e municipais, da administração direta e indireta e das fundações mantidas pelo poder público.

Diante disse aspecto apresentado, relativo ao SUS, é importante destacar a participação do profissional de Educação Física como membro de equipes multiprofissionais que atuam, principalmente, nos Núcleos de Apoio à Saúde da Família - NASF, núcleos estes que são constituídos por equipes compostas por profissionais de diversas áreas de conhecimento, visando contribuir para a integralidade do cuidado aos usuários do SUS (BRASIL, 2011).

\section{Aspectos epidemiológicos e econômicos}

Luna et al. (2013) afirmam que o acompanhamento epidemiológico das doenças, feito através de sistemas de vigilância em saúde pública, possibilita a detecção precoce de surtos e epidemias, além de recomendarem medidas de prevenção e controle dessas doenças.

Por meio desses sistemas de vigilância em saúde é possível identificar, também, quais as doenças crônicas não transmissíveis são mais recorrentes, as regiões de abrangência e os fatores de risco que contribuem para sua existência. Com esses dados, há possibilidades de se elaborar propostas de intervenção pertinentes a fim de prevenir e/ou controlar essas doenças.

O Ministério da Saúde afirma que as doenças crônicas não transmissíveis são um dos maiores problemas de saúde pública da atualidade. Malta et al. (2013) explicam que elas estão relacionadas com elevado número de mortes prematuras, perda da qualidade de vida, com alto grau de limitação e incapacidade, além de impactos econômicos para famílias, comunidades e a sociedade em geral. Em 2016 as doenças crônicas não transmissíveis foram responsáveis por $74 \%$ (setenta e quatro por cento) do total de mortes no Brasil, destacando-se as doenças cardiovasculares $(28 \%)$, as neoplasias $(18 \%)$, as doenças respiratórias $(6 \%)$ e o diabetes (5\%) (BRASIL, 2019). 
Não é novidade dizer que existem várias evidências de que ações integradas de promoção da saúde diminuam determinados percentuais de doenças, agindo diretamente nos fatores de risco relacionados a elas. Dentre os principais fatores de risco pontuados pelo Ministério de Saúde, estão: tabagismo, consumo abusivo de bebida alcoólica, inatividade física e excesso de peso e obesidade.

No que se refere aos gastos relacionados com saúde pública no Brasil, é possível verificarmos, a partir dos dados publicados pelo Ministério da Saúde, por meio do Fundo Nacional de Saúde, que a atenção de média e alta complexidade laboratorial e hospitalar têm impacto financeiro extremamente alto (de 2014 a 2018, oscilou de 40 a 50 bilhões de reais gastos por ano), investimentos estes que podem ser amenizados a partir de políticas públicas de prevenção à doenças e promoção da saúde eficazes. A Atenção Básica também se mostra dispendiosa. Em 2018 investiu mais de 20 bilhões de reais.

\section{O panorama sobre o profissional de Educação Física na saúde pública: análises e discussões}

Santos e Benedetti (2012), ao analisarem a inserção do profissional de Educação Física nos Núcleos de Apoio à Saúde da Família NASF, verificaram que a Educação Física está inserida em 49,2\% das equipes de NASF. Porém, o coeficiente de profissional por população coberta pela Estratégia Saúde da Família, foi de aproximadamente 1 (um) para 100.000 (cem mil) pessoas, que dificulta a assistência pública em saúde por parte deste profissional.

Os autores creem, ainda, que com a abertura de uma associação que apoie a implementação e desenvolvimento de cursos de Educação Física com eixo na integralidade, na formação e na atenção à saúde, tendo como preocupação a perícia técnico-científica e saberes sócio-políticos que garantam mais segurança para a empregabilidade e resolução de problemas no setor de interesse do profissional; serão formados profissionais de Educação Física com competência técnica para ajudar na consolidação do atual sistema público de saúde.

Ao perceberem, também, a frágil formação do bacharel em Educação Física para atuar na saúde pública, Falci e Belisário (2013) sugerem que a formação seja complementada por curso(s) de pós-graduação, visto ter um enfoque multidisciplinar, que é necessário para a formação dos profissionais desta área. 
Em seus estudos, Florindo (2009) aponta que o entendimento errôneo dos objetivos dos Núcleos de Apoio à Saúde da Família - núcleos, estes, que surgiram para melhorar o atendimento na atenção básica na Estratégia Saúde da Família e auxiliar na Política Nacional de Promoção da Saúde - é frequente entre os profissionais que integram as equipes multiprofissionais.

Além disso, o autor comenta que existem questões como a insistência na ênfase em trabalhos individuais em detrimento aos trabalhos coletivos e a falta de estratégias para a atuação no âmbito coletivo, que, a nosso ver, podem interferir de forma negativa na eficiência e qualidade das ações das equipes. Mesmo não citando somente o profissional de Educação Física, é notório que este, como membro de equipe multiprofissional dos Núcleos de Apoio à Saúde da Família, deva ser considerado.

$\mathrm{Na}$ direção desse pensamento, verificamos que Loch e Florindo (2012) abordam questões sobre a composição da Educação Física nas residências multiprofissionais na área da saúde, afirmando que a necessária aproximação entre o "mundo acadêmico da Educação Física e atuação profissional" pode ocorrer porque "as residências são espaços onde serviço e ensino estão intimamente ligados. Os autores dizem que a solicitação para que a Educação Física participe dessas residências só não é maior porque ainda existe descrença quanto à formação inicial para a atuação no contexto da atenção primária. Eles enfatizam, ainda, que é compreensível essa descrença pois, mesmo tendo melhorado nos últimos anos, a formação em Educação Física tem abordado as questões relacionadas à Saúde Pública de maneira insuficiente.

A pesquisa de Andrello et al. (2012) teve como objetivo investigar a percepção dos secretários de saúde dos principais municípios da região norte do Paraná sobre a importância da atividade física nas políticas de saúde e observou-se uma visão restrita, como se as atividades realizadas por este profissional devessem ser exatamente as mesmas das desenvolvidas em outros contextos, como em academias de ginástica, por exemplo. Os autores mencionam que apesar da dinamicidade do campo político, é possível que secretários municipais de saúde de outras regiões do país apresentem a mesma visão acerca da atuação do profissional de Educação Física no âmbito da saúde pública.

Foi possível constatar nos estudos de Carvalho et al (2017) que esta atuação acontece, por vezes, de maneira limitada. Com a análise de vários registros, os autores notaram que a atuação deste profissional está voltada a, apenas, remediar os efeitos de uma vida sedentária. Foi exposto pelos teóricos, ainda, que a maioria das intervenções se apresenta em 
caráter curativo e trata, principalmente, sobre aspectos relacionados ao público idoso.

Scabar et al. (2012), sugerem que haja mais incentivo à implementação das ideias de promoção da saúde na área da Educação Física, de modo a permitir que o profissional deste setor obtenha um perfil que o permita adotar posturas condizentes ao conceito de promoção da saúde, com base em um trabalho social e inclusivo, para que o indivíduo seja pensado em sua integralidade e não de forma fragmentada.

Ao realizar uma pesquisa com egressos de um curso de bacharel em Educação Física, no que diz respeito à sua inserção no mercado de trabalho na perspectiva do Sistema Único de Saúde, Candido et al. (2018) verificaram a baixa representatividade de egressos nesse setor, observando que, dos quinze voluntários, apenas dois estavam inseridos na área da saúde pública.

Neves et al. (2015) contribuem no sentido de afirmar que para que o profissional de Educação Física ganhe espaço no Sistema Único de Saúde - SUS, este deve entender como funciona a gestão pública, as contradições, disputas, dificuldades e desafios dos trabalhos realizados no SUS, levando em consideração os usuários sobre as atividades de Educação Física, os profissionais de saúde e as representações sociais de gestores.

Ficou evidente que mesmo com os avanços ocorridos nos últimos anos, a inserção do profissional de Educação Física no campo da saúde pública, considerando as diretrizes do Sistema Único de Saúde, deve ser abordada de forma mais técnica durante a formação acadêmica.

$\mathrm{Na}$ formação do profissional multidisciplinar, no caso o Profissional de Educação Física, as questões precisam ser trabalhadas para além dos conhecimentos fisio-biológicos, de maneira que tal formação seja construída com subsídios teóricos e práticos tenha bagagem suficiente para ingressar nesse setor de maneira ampliada e satisfatória (para ele, para os usuários, para a consolidação do sistema de saúde atual).

\section{Considerações finais}

Apesar da tímida quantidade de artigos científicos que tratam pontos sobre o papel do profissional de Educação Física no âmbito da saúde pública, há uma crescente preocupação com relação à inserção deste profissional nesta área, tendo em vista sua capacitação como especialista em atividades e exercícios físicos, capaz de promover a saúde e o 
bem-estar através de atividades que objetivem recuperar e manter a saúde e prevenir doenças.

Durante o período de dez anos (2009 a 2019) o que mais foi discutido sobre o profissional de Educação Física, no que diz respeito à sua atuação na saúde pública, foi: dificuldades de adaptação à área da saúde mesmo com formação de bacharel; formação acadêmica não condizente com a prática profissional; dificuldade de trabalhar em equipes multiprofissionais; e a pouca demanda de egressos que partem para a área da saúde pública, prevalecendo a adesão às outras áreas de atuação (fitness e de competição).

É redundante afirmar a importância do trabalho desse profissional nos ambientes de saúde pública. Mas, vale destacar que sua inserção, desde seu reconhecimento como ator do campo da saúde, até os dias atuais, vem sendo explorado e (re)qualificado estrategicamente a fim de atender às demandas dos usuários da rede pública de saúde, bem como auxiliar na consolidação do sistema atual.

Com esta pesquisa, foi possível conhecer os principais entraves que, possivelmente, limitam a demanda de profissionais para essa área. Ficou evidente que a inserção do profissional de Educação Física na saúde pública vem sendo feita de acordo com as implantações de programas, como a Estratégia Saúde da Família, Centros de Atendimento Psicossociais e Núcleos de Apoio à Saúde da Família, como prevê a legislação. Porém, sua atuação ainda é limitada e, podemos dizer, intimidada, principalmente pela insegurança de atuar com equipes multiprofissionais e pela dificuldade em atender os usuários de forma integral (sem fragmentá-lo).

Podemos concluir que devemos buscar melhorias para a atuação do profissional de Educação Física na saúde pública, por meio de metodologias de formação eficazes (tanto à nível de graduação, quanto de pós-graduação). No mesmo ritmo, gestores da área devem dar espaço e autonomia para que o profissional em questão possa atuar de forma condizente com as demandas da sociedade.

\section{Referências}

AIRES, Margarida de Mello. Fisiologia. - 5. ed. - Rio de Janeiro: Guanabara Koogan, 2018.

ANDRELLO, Eduardo; LOCH, Mathias Roberto; JUNIOR, Anísio Calciolari; REICHERT, Felipe Fossati. Atividade Física e saúde pública sob o olhar de se- 
cretários municipais de saúde. Revista Brasileira de Atividade Física \& Saúde. Pelotas/RS, v.17, n. 3, p.206-211, jun/2012.

ASSIS, Mônica de. Promoção da saúde e envelhecimento: orientações para o desenvolvimento de ações educativas com idosos. Rio de Janeiro, CRDE UnATI UERJ, 2002.

BARRET, Kim E. et al. Fisiologia médica de Ganong [recurso eletrônica]; [tradução Ademar Valadares Fonseca, Geraldo Serra, Luís Fernando Marques Dorivllé; revisão técnica: Luciano Stürmer de Fraga, Renata Padilha Guedes]. 24. ed. - Dados eletrônicos. - Porto Alegre: AMGH, 2014.

BRASIL. Ministério da Saúde. Fundo Nacional de Saúde. Gráfico comparativo por ano. Comparativo de recursos repassados por ano (2018 a 2019-até a presente data). Disponível em < $\underline{\text { https://consultafns.saude.gov.br/\#/compara- }}$ tivo >. Acesso em: 29 nov. 2019.

- Ministério da Saúde. Secretaria de Vigilância em Saúde. Departamento de Análise em Saúde e Vigilância de Doenças não Transmissíveis. Vigitel Brasil 2018: vigilância de fatores de risco e proteção para doenças crônicas por inquérito telefônico: estimativas sobre frequência e distribuição sociodemográfica de fatores de risco e proteção para doenças crônicas nas capitais dos 26 estados brasileiros e no Distrito Federal em 2018 / Brasília: Ministério da Saúde, 2019.

Ministério da Saúde. Portaria n. ${ }^{\circ}$ 2.488, de 21 de outubro de 2011: Aprova a Política Nacional de Atenção Básica e estabelece a revisão de diretrizes e normas para a organização da Atenção Básica, para a Estratégia Saúde da Família e o Programa de Agentes Comunitários de Saúde. Disponível em: <www.bvsms.saude.gov.br/bvs/saudelegis/gm/2011/ prt2488_21_10_2011.html>. Acesso em: 28 nov. 2019.

CANDIDO, Luana de Oliveira; ROSSIT, Rosana Aparecida Salvador; OLIVEIRA, Rogério Cryz de. Inserção profissional dos egressos de um curso de Educação Física com ênfase na formação em saúde. Trab. Educ. Saúde, Rio de Janeiro, v. 16, n. ${ }^{\circ}$ 1, p. 305-318, jan/abr 2018.

CARVALHO, Anderson dos Santos; ABDALLA, Pedro Pugliesi; JÚNIOR, Carlos Roberto Bueno. Atuação do profissional de Educação Física no Sistema Único de Saúde: revisão sistemática. Revista Brasileira de Promoção da Saúde, Fortaleza, v.30, n. 3, p.1-11, jul/set 2017.

CARVAlHO, Gilson. Saúde Pública. Estudos Avançados, 27(78), 2013.

DÂMASO, Ana. R.; SANCHES, Priscila de Lima. Exercício Físico no Tratamento da Obesidad I: como prescrever? In: MANCINI, Marcio C. [et al]. Tratado de Obesidade - 2. ed. - Rio de Janeiro: Guanabara Koogan, 2015.

FALCI, Denise Mourão; BELISÁRIO, Soraya Almeida. A inserção do profissional de Educação Física na Atenção Primária à Saúde e os desafios em sua for- 
mação. Interface Comunicação Saúde e Educação, v. 17, n. 47, p. 885-99, out/dez 2013.

FERNANDES, Ana Mércia Barbosa Leite et al. Efeitos da prática de exercício físico sobre o desempenho da marcha e da mobilidade funcional em idosos. Fisioter Mov. 2012 out/dez; 25(4): 821-30

FERNANDES, Gonçalo et al. O contributo da Educação Física para o desenvolvimento motor: uma revisão sistemática. Gymnasium, v.2, n.2: p.1-6, 2017 FLORINDO, Alex Antônio. Núcleos de Apoio à Saúde da Família e a Promoção das Atividades Físicas no Brasil: de onde viemos, onde estamos e para onde vamos. Revista Brasileira de Atividade Física \& Saúde. Vol. 14, Número 1, 2009.

GALLAHUE, David L. Compreendendo o desenvolvimento motor [recurso eletrônico]: bebês, crianças, adolescentes e adultos / David L. Gallahue, John C. Ozmun, Jaqueline D. Goodway; tradução: Denise Regina de Sales; revisão técnica: Ricardo D. S. Petersen. - 7. ed. - Dados eletrônicos. - Porto Alegre: AMGH, 2013.

GREGUOL, Márcia; COSTA, Roberto Fernandes da (organizadores). Atividade Física Adaptada: qualidade de vida para pessoas com necessidades especiais. - 3. ed. rev. e ampl. - Barueri, SP: Manole, 2013.

LOCH, Mathias Roberto; FLORINDO, Alex Antonio. A Educação Física e as residências multiprofissionais em saúde. Revista Brasileira de Atividade Física \& Saúde. Pelotas. v.17, n.1, abr/2012.

LUNA, Expedito José de Albuquerque; ARAÚJO, Wildo Navegantes de; CAVALCANTI, Luciano Pamplona de Góes. Vigilância Epidemiológica. In: ROUQUAYROL, Maria Zélia; SILVA, Marcelo Gurgel Carlos da. Epidemiologia \& Saúde. - 7. ed. - Rio de Janeiro: MedBook, 2013.

MALTA, Deborah Carvalho; MOURA, Lenildo de; SILVA JÚNIOR, Jarbas Barbosa da; Epidemiologia das doenças crônicas não transmissíveis no Brasil. In: ROUQUAYROL, Maria Zélia; SILVA, Marcelo Gurgel Carlos da. Epidemiologia \& Saúde. - 7. ed. - Rio de Janeiro: MedBook, 2013.

MANCINI, Marcio C. Obesidade e doenças associadas. In: MANCINI, Marcio C. ... [et al]. Tratado de Obesidade - 2. ed. - Rio de Janeiro: Guanabara Koogan, 2015.

MEREGE FILHO, Carlos Alberto Abujabra et al. Associação entre o nível de atividade física de lazer e o desempenho cognitivo em crianças saudáveis. Rev Bras Educ Fís Esporte, São Paulo, v. 27, n.3, p. 355-61, jul-set, 2013

NASCIMENTO, Simony Lira do; GODOY, Ana Carolina; SURITA, Fernanda Garnhani; SILVA, João Luiz Pinto e. Recomendações para a prática de exercí- 
cio físico na gravidez: uma revisão crítica da literatura. Rev Bras Ginecol Obstet. v. 36, n. 9, p.423-31, 2014.

NEVES, Ricardo Lira de Rezende; ANTUNES, Priscilla de Cesaro; BAPTISTA, Tadeu João Ribeiro; ASSUMPÇÃO, Luís Otávio Teles. Educação Física na Saúde Pública: revisão sistemática. Rev Bras Ci. e Mov. v. 23, n. 2, p.163-177, 2015

NUNES, Marcello Pereira; VOTRE, Sebastião Josué; SANTOS, Wagner dos. $O$ profissional de educação física no Brasil: desafios e perspectivas no mundo do trabalho. Motriz, Rio Claro, v. 18 n. 2, p. 280-290, abr/jun. 2012

PEDRINELLI, Verena Junghähnel; VERENGUER, Rita de Cássia Garcia. Educação Física Adaptada: introdução ao universo das possibilidades. In: GREGUOL, Márcia; COSTA, Roberto Fernandes da (organizadores). Atividade Física Adaptada: qualidade de vida para pessoas com necessidades especiais. - 3. ed. rev. e ampl. - Barueri, SP: Manole, 2013.

ROSEMBERG, Ana Margarida Furtado Arruda. Breve História da Saúde Pública no Brasil. In: ROUQUAYROL, Maria Zélia; SILVA, Marcelo Gurgel Carlos da. Epidemiologia \& Saúde. - 7. ed. - Rio de Janeiro: MedBook, 2013.

SALLES, William das Neves; FARIAS, Gelcemar Oliveira; NASCIMENTO, Juarez Vieira do. Inserção profissional e formação continuada de egressos de curso de graduação em Educação Física. Rev Bras Educ Fís Esporte, São Paulo, v.29, n. 3, p.475-86, jul-set 2015

SANTOS, Sueyla F. da Silva dos. BENEDETTI, Tânia R. Bertoldo. Cenário de implantação do Núcleo de Apoio à Saúde da Família e a inserção do profissional de Educação Física. Rev Bras Ativ Fís e Saúde. Pelotas/RS, v.17, n.3, p. 188-194, jun/2012.

SANTOS, Lenir. Sistema Único de Saúde: os desafios da gestão interfederativa; revisão: Olivia Yumi. - Campinas, SP: Saberes Editora, 2013.

SCABAR, Thaís Guerreiro; PELICIONI, Andrea Focesi; PELICIONI, Maria Cecília Focesi. Atuação do profissional de Educação Física no Sistema Único de Saúde: uma análise a partir da Política Nacional de Promoção da Saúde e das Diretrizes do Núcleo de Apoio à Saúde da Família - NASF. J Health Sci Inst. v.30, n.4, p.411-8, 2012.

SILVA, Erica Natalia Alves de Lima da; LUCENA, Rogério Rocha. A prática de exercício físico na gestação. UNAERP. Revista Científica Integrada, v. 2, ed. 4. 2014. Disponível em <https://www.unaerp.br/revista-cientifica-integrada/edicoes-anteriores/volume-2-edicao-4/2048-a-pratica-de-exercicio-fisicona-gestacao/file>. Acesso em: 19 nov. 2019.

SOUZA, Thays Kéllen Rodrigues de; VIEIRA, Raquel de Paula; NETO, Antônio Alves Leite. O desenvolvimento fetal influenciado pela prática de exercício 
físico por gestantes. Caderno de Resumos Expandidos Internos, v. 1, n. 1, 2018.

VIVOLO, Sandra Roberta G. Ferreira; SARNO, Flávio. Epidemiologia da obesidade em adultos no Brasil e no mundo. In: MANCINI, Marcio C. [et al]. Tratado de Obesidade - 2. ed. - Rio de Janeiro: Guanabara Koogan, 2015. 
